\title{
High-resolution Anorectal Manometry and Anal Endosonographic Findings in the Evaluation of Fecal Incontinence
}

\author{
Tae Hee Lee and Joon Seong Lee*
}

Institute for Digestive Research, Soonchunhyang University, College of Medicine, Seoul, Korea

A 65-year-old woman presented with moderate volume of fecal incontinence during the past 2 years. She usually was not aware of it although sometimes she was not able to hold back stool until going to the bathroom in time. The stools were usually soft and formed. She denied neurologic symptoms, urinary incontinence and pelvic or perianal injury. She had 2 children by natural childbirth. She has taken nonsteroidal anti-inflammatory drug for osteoarthritis. Perianal examination showed unremarkable

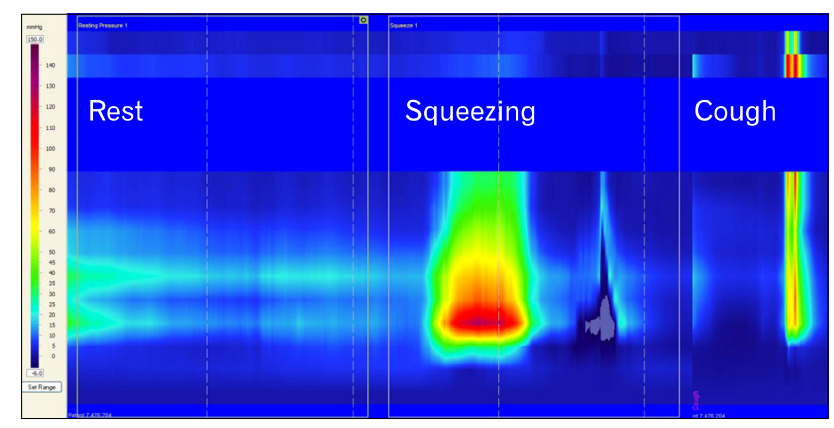

Figure 1. The low mean resting anal pressure $(28 \mathrm{mmHg})$, maximum squeezing pressure $(135 \mathrm{mmHg})$ and short duration of sustained squeezing pressure ( 5 second) are observed. During coughing, abrupt increase of anal sphincter pressure is noted. findings. Digital rectal examination revealed weak resting tone and normal increase with squeeze. However, digital rectal examination is not reliable and is subject to interobserver differences due to several factors including the size of the examiner's finger, the technique and the cooperation of patient. ${ }^{1}$ Therefore high-resolution anorectal manometry (ManoScan, Sierra Scienti-

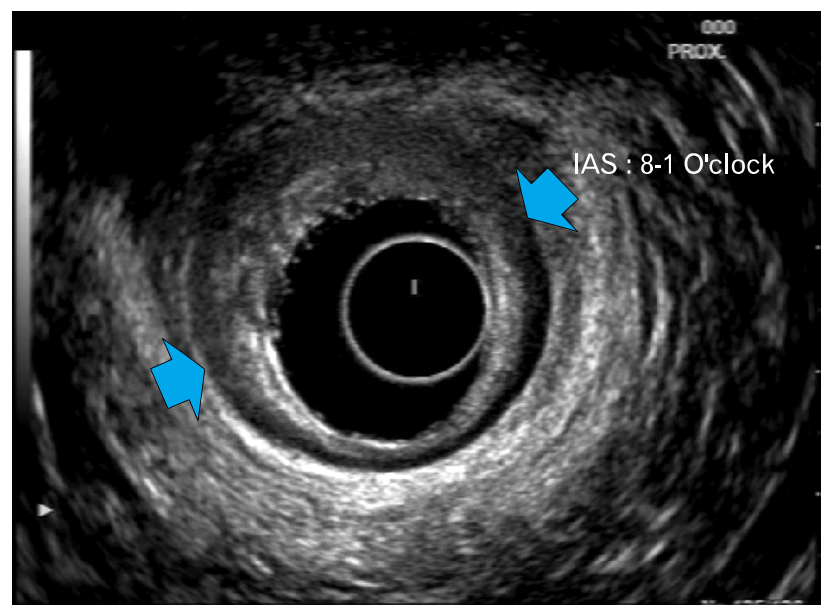

Figure 2. Anal endosonography shows the presence of scarring of internal anal sphincter from the 8 to 1 -o'clock direction.

Received: August 7, 2012 Revised: August 9, 2012 Accepted: August 10, 2012

(C) This is an Open Access article distributed under the terms of the Creative Commons Attribution Non-Commercial License (http://creativecommons. org/licenses/by-nc/3.0) which permits unrestricted non-commercial use, distribution, and reproduction in any medium, provided the original work is properly cited.

*Correspondence: Joon Seong Lee, MD, PhD Institute for Digestive Research, Soonchunhyang University, College of Medicine, Daesagwan-gil 22, Yongsan-gu, Seoul 140-743, Korea

Tel: +82-2-709-9691, Fax: +82-2-709-9696, E-mail: drjslee@dreamwiz.com

Financial support: None. Conflicts of interest: None. 
fic Instruments, Los Angeles, CA, USA) and anal endosonography were performed. The high-resolution anorectal manometry showed a very low mean resting anal pressure, relatively intact maximal squeezing pressure and short duration of the sustained squeezing pressure. However abrupt increase of anal sphincter pressure above rectal pressure that could prevent stress incontinence was observed during cough (Fig. 1). Because resting anal pressure predominantly represents the internal anal sphincter (IAS) pressure and the squeezing pressure predominantly measures the external anal sphincter pressure, these findings imply defect in IAS with normal external anal sphincter. ${ }^{2}$
The anal endosonography also revealed the presence of scarring of IAS from the 8 to 1-o'clock direction (Fig. 2). In this patient, fecal incontinence was turned out to be caused by the IAS defect.

\section{References}

1. Rao SS, American College of Gastroenterology Practice Parameters Committee. Diagnosis and management of fecal incontinence. American College of Gastroenterology Practice Parameters Committee. Am J Gastroenterol 2004;99:1585-1604.

2. Maslekar S, Gardiner A, Maklin C, Duthie GS. Investigation and treatment of faecal incontinence. Postgrad Med J 2006;82:363-371. 